\title{
Padé Approximant for the Equation of Motion of a Supernova Remnant
}

\author{
Lorenzo Zaninetti \\ Physics Department, via P. Giuria 1, Turin, Italy \\ Email: zaninetti@ph.unito.it
}

How to cite this paper: Zaninetti, L. (2017) Padé Approximant for the Equation of Motion of a Supernova Remnant. Journal of High Energy Physics, Gravitation and Cosmology, 3, 78-86.

http://dx.doi.org/10.4236/jhepgc.2017.31011

Received: October 7, 2016

Accepted: December 25, 2016

Published: December 28, 2016

Copyright $\odot 2017$ by author and Scientific Research Publishing Inc. This work is licensed under the Creative Commons Attribution International License (CC BY 4.0).

http://creativecommons.org/licenses/by/4.0/

\begin{abstract}
In this paper we derive three equations of motion for a supernova remnant (SNR) in the framework of the thin layer approximation using the Padé approximant. The circumstellar medium is assumed to follow a density profile of either an exponential type, a Gaussian type, or a Lane-Emden $(n=5)$ type. The three equations of motion are applied to four SNRs: Tycho, Cas A, Cygnus loop, and SN 1006. The percentage error of the Padé approximated solution is always less than $10 \%$. The theoretical decrease of the velocity over ten years for SNRs is evaluated.
\end{abstract}

\section{Keywords}

Supernovae: General, ISM: Supernova Remnants, Supernovae: Individual (Tycho), Supernovae: Individual (Cas A), Supernovae: Individual (Cygnus Loop),

Supernovae: Individual (SN 1006)

\section{Introduction}

The equation of motion for a supernova remnant (SNR) can be modeled by a single law of motion or multiple laws of motion when the appropriate boundary conditions are provided. Examples of a single law of motion are: The Sedov expansion in the presence of a circumstellar medium (CSM) with constant density where the radius, $r$, scales as $r \propto t^{0.4}$, see [1], and the momentum conservation in the framework of the thin layer approximation with CSM at constant density where $R \propto t^{0.25}$, see [2]. Examples of piece-wise solutions for an SNR can be found in [3]: A first energy conserving phase, $r \propto t^{0.4}$ followed by a second adiabatic phase where $r \propto t^{0.285}$. At the same time it has been shown that in the first ten years of SN 1993J 1993j $r \propto t^{0.82}$, which means an observed exponent larger than the previously suggested exponents, see [4]. The previous analysis allows posing a basic question: "Is it possible to find an analytical solution for SNRs given the three observable astronomical parameters, age, radius and velocity ?". In order to answer the above question, Section 2 introduces three profiles 
for the CSM, Section 3 derives three Padé approximated laws of motion for SNRs, and Section 4 closes the derived equations of motion for four SNRs.

\section{Profiles of Density}

This section introduces three density profiles for the CSM: An exponential profile, a Gaussian profile, and a self-gravitating profile of Lane-Emden type.

\subsection{The Exponential Profile}

This density is assumed to have the following exponential dependence on $r$ in spherical coordinates:

$$
\rho\left(r ; r_{0}, b, \rho_{0}\right)=\rho_{0} \exp \left(-\frac{\left(r-r_{0}\right)}{b}\right),
$$

where $b$ represents the scale. The piece-wise density is

$$
\rho\left(r ; r_{0}, b, \rho_{0}\right)= \begin{cases}\rho_{0} & \text { if } r \leq r_{0} \\ \rho_{0} \exp \left(-\frac{\left(r-r_{0}\right)}{b}\right) & \text { if } r>r_{0}\end{cases}
$$

The total mass swept, $M\left(r ; r_{0}, b, \rho_{0}\right)$, in the interval $[0, r]$ is

$$
\begin{aligned}
M\left(r ; r_{0}, b, \rho_{0}\right)= & \frac{4}{3} \rho_{0} \pi r_{0}^{3}-4 b\left(2 b^{2}+2 b r+r^{2}\right) \rho_{0} \mathrm{e}^{\frac{r_{0}-r}{b}} \pi \\
& +4 b\left(2 b^{2}+2 b r_{0}+r_{0}^{2}\right) \rho_{0} \pi .
\end{aligned}
$$

\subsection{The Gaussian Profile}

This density has the Gaussian dependence

$$
\rho\left(r ; r_{0}, b, \rho_{0}\right)=\rho_{0} \exp \left(-\frac{1}{2} \frac{r^{2}}{b^{2}}\right),
$$

and the piece-wise density is

$$
\rho\left(r ; r_{0}, b, \rho_{0}\right)= \begin{cases}\rho_{0} & \text { if } r \leq r_{0} \\ \rho_{0} \exp \left(-\frac{1}{2} \frac{r^{2}}{b^{2}}\right) & \text { if } r>r_{0}\end{cases}
$$

The total mass swept, $M\left(r ; r_{0}, b, \rho_{0}\right)$, in the interval $[0, r]$ is

$$
\begin{aligned}
M\left(r ; r_{0}, b, \rho_{0}\right)= & \frac{4}{3} \rho_{0} \pi r_{0}^{3}+4 \rho_{0} \pi\left(-\mathrm{e}^{-\frac{1 r^{2}}{2 b^{2}}} r b^{2}+\frac{1}{2} b^{3} \sqrt{\pi} \sqrt{2} \operatorname{erf}\left(\frac{1}{2} \frac{\sqrt{2} r}{b}\right)\right) \\
& -4 \rho_{0} \pi\left(-\mathrm{e}^{-\frac{1 r_{0}^{2}}{2 b^{2}}} r_{0} b^{2}+\frac{1}{2} b^{3} \sqrt{\pi} \sqrt{2} \operatorname{erf}\left(\frac{1}{2} \frac{\sqrt{2} r_{0}}{b}\right)\right),
\end{aligned}
$$

where erf is the error function, see [5].

\subsection{The Lane-Emden Profile}

The Lane-Emden profile when $n=5$, after [6] [7], is 


$$
\begin{gathered}
\rho\left(r ; r_{0}, b, \rho_{0}\right)=\rho_{0} \frac{1}{\left(1+\frac{r^{2}}{3 b^{2}}\right)^{\frac{5}{2}}}, \\
\rho\left(r ; r_{0}, b, \rho_{0}\right)= \begin{cases}\rho_{0} & \text { if } r \leq r_{0} \\
\rho_{0} \frac{1}{\left(1+\frac{r^{2}}{3 b^{2}}\right)^{\frac{5}{2}}} & \text { if } r>r_{0}\end{cases}
\end{gathered}
$$

The total mass swept, $M\left(r ; r_{0}, b, \rho_{0}\right)$, in the interval $[0, r]$ is

$$
M\left(r ; r_{0}, b, \rho_{0}\right)=\frac{4}{3} \rho_{0} \pi r_{0}^{3}+4 \frac{b^{3} r^{3} \rho_{0} \sqrt{3} \pi}{\left(3 b^{2}+r^{2}\right)^{\frac{3}{2}}}-4 \frac{b^{3} r_{0}^{3} \rho_{0} \sqrt{3} \pi}{\left(3 b^{2}+r_{0}^{2}\right)^{\frac{3}{2}}} .
$$

\section{The Equation of Motion}

The conservation of the momentum in spherical coordinates in the framework of the thin layer approximation states that

$$
M_{0}\left(r_{0}\right) v_{0}=M(r) v
$$

where $M_{0}\left(r_{0}\right)$ and $M(r)$ are the masses swept at $r_{0}$ and $r$, and $v_{0}$ and $v$ are the velocities of the thin layer at $r_{0}$ and $r$.

\subsection{Motion with Exponential Profile}

Assuming an exponential profile as given by Equation (2) the velocity is

$$
\frac{\mathrm{d} r}{\mathrm{~d} t}=\frac{N E}{D E},
$$

where

$$
N E=-r_{0}^{3} v_{0},
$$

and

$$
D E=6 \mathrm{e}^{\frac{r_{0}-r}{b}} b^{3}+6 \mathrm{e}^{\frac{r_{0}-r}{b}} b^{2} r+3 \mathrm{e}^{\frac{r_{0}-r}{b}} b r^{2}-r_{0}^{3}-3 r_{0}^{2} b-6 r_{0} b^{2}-6 b^{3} .
$$

In the above differential equation of the first order in $r$, the variables can be separated and integration gives the following non-linear equation:

$$
\begin{aligned}
& \frac{1}{r_{0}^{3} v_{0}}\left(18 \mathrm{e}^{\frac{r_{0}-r}{b}} b^{4}+12 \mathrm{e}^{\frac{r_{0}-r}{b}} b^{3} r+3 \mathrm{e}^{\frac{r_{0}-r}{b}} b^{2} r^{2}-r_{0}^{4}-3 r_{0}^{3} b\right. \\
& \left.+r_{0}^{3} r-9 r_{0}^{2} b^{2}+3 r_{0}^{2} b r-18 b^{3} r_{0}+6 r_{0} b^{2} r-18 b^{4}+6 b^{3} r\right) \\
& =\left(t-t_{0}\right) .
\end{aligned}
$$

In this case is not possible to find an analytical solution for the radius, $r$, as a function of time. We therefore apply the Padé rational polynomial approximation of degree 2 in the numerator and degree 1 in the denominator about the point $r=r_{0}$ to the left-hand side of Equation (12):

$$
\frac{-\left(r_{0}-r\right)\left(-5 b r-b r_{0}-2 r r_{0}+2 r_{0}^{2}\right)}{2 v_{0}\left(2 b r-5 b r_{0}-r r_{0}+r_{0}^{2}\right)}=t-t_{0} .
$$


The resulting Padé approximant for the radius $r_{2,1}$ is

$$
\begin{aligned}
r_{2,1}= & \frac{1}{2 r_{0}+5 b}\left(r_{0} t v_{0}-r_{0} t_{0} v_{0}-2 b t v_{0}+2 b t_{0} v_{0}+2 r_{0}^{2}+2 r_{0} b\right. \\
& +\left(4 b^{2} t^{2} v_{0}^{2}-8 b^{2} t t_{0} v_{0}^{2}+4 b^{2} t_{0}^{2} v_{0}^{2}-4 b t^{2} r_{0} v_{0}^{2}+8 b t t_{0} r_{0} v_{0}^{2}\right. \\
& -4 b t_{0}^{2} r_{0} v_{0}^{2}+t^{2} r_{0}^{2} v_{0}^{2}-2 t t_{0} r_{0}^{2} v_{0}^{2}+t_{0}^{2} r_{0}^{2} v_{0}^{2}+42 b^{2} t r_{0} v_{0} \\
& \left.\left.-42 b^{2} t_{0} r_{0} v_{0}+6 b t r_{0}^{2} v_{0}-6 b t_{0} r_{0}^{2} v_{0}+9 r_{0}^{2} b^{2}\right)^{\frac{1}{2}}\right)
\end{aligned}
$$

and the velocity is

$$
\begin{gathered}
v_{2,1}=\frac{\mathrm{d} r_{2,1}}{\mathrm{~d} t}=\frac{N V E}{D V E}, \\
N V E=4 v_{0}\left\{\left(-b / 2+1 / 4 r_{0}\right) \times \sqrt{4\left(b-\frac{1}{2} r_{0}\right)^{2}\left(t-t_{0}\right)^{2} v_{0}^{2}+42\left(b+1 / 7 r_{0}\right)\left(t-t_{0}\right) b r_{0} v_{0}+9 r_{0}^{2} b^{2}}\right. \\
\left.+\left(3 / 4 b+\left(t / 4-1 / 4 t_{0}\right) v_{0}\right) r_{0}^{2}+\frac{21 r_{0} b}{4}\left(v_{0}\left(-\frac{4 t}{21}+\frac{4 t_{0}}{21}\right)+b\right)+b^{2} v_{0}\left(t-t_{0}\right)\right\},
\end{gathered}
$$

and

$$
D V E=\sqrt{4\left(b-\frac{1}{2} r_{0}\right)^{2}\left(t-t_{0}\right)^{2} v_{0}^{2}+42\left(b+1 / 7 r_{0}\right)\left(t-t_{0}\right) b r_{0} v_{0}+9 r_{0}^{2} b^{2}} \times\left(2 r_{0}+5 b\right) .
$$

\subsection{Motion with Gaussian Profile}

Assuming a Gaussian profile as given by Equation (4) the velocity is

$$
\frac{\mathrm{d} r}{\mathrm{~d} t}=\frac{N G}{D G},
$$

where

$$
N G=-2 r_{0}^{3} v_{0}
$$

and

$$
\begin{aligned}
D G= & -3 b^{3} \sqrt{\pi} \sqrt{2} \operatorname{erf}\left(\frac{1}{2} \frac{\sqrt{2} r}{b}\right)+3 b^{3} \sqrt{\pi} \sqrt{2} \operatorname{erf}\left(\frac{1}{2} \frac{\sqrt{2} r_{0}}{b}\right) \\
& +6 \mathrm{e}^{-\frac{1}{2} r^{2}} r b^{2}-6 \mathrm{e}^{-\frac{1}{2} r_{0}^{2}} r_{0} b^{2}-2 r_{0}^{3} .
\end{aligned}
$$

The appropriate non-linear equation is

$$
\begin{aligned}
& \frac{1}{2 r_{0}^{3} v_{0}}\left(\left(-12 b^{4}+6 r_{0}\left(r-r_{0}\right) b^{2}\right) \mathrm{e}^{-\frac{1 r_{0}^{2}}{2} b^{2}}+12 b^{4} \mathrm{e}^{-\frac{1}{2} r^{2}}\right. \\
& \left.-3 \sqrt{\pi} \operatorname{erf}\left(\frac{1}{2} \frac{\sqrt{2} r_{0}}{b}\right) \sqrt{2} b^{3} r+3 b^{3} \sqrt{\pi} \sqrt{2} \operatorname{erf}\left(\frac{1}{2} \frac{\sqrt{2} r}{b}\right) r+2 r_{0}^{3}\left(r-r_{0}\right)\right) \\
& =t-t_{0} .
\end{aligned}
$$

The Padé rational polynomial approximation of degree 2 in the numerator and degree 1 in the denominator about $r=r_{0}$ for the left-hand side of the above equation gives 


$$
\frac{1}{2 v_{0}\left(2 b^{2} r-5 r_{0} b^{2}-r r_{0}^{2}+r_{0}^{3}\right)}\left(-\left(r-r_{0}\right)\left(9 \mathrm{e}^{-\frac{1}{2} b_{0}^{2}} b^{2} r-9 \mathrm{e}^{-\frac{1}{2} b_{0}^{2}} r_{0} b^{2}-4 b^{2} r+10 r_{0} b^{2}+2 r r_{0}^{2}-2 r_{0}^{3}\right)\right)=t-t_{0} \text {. }
$$

The resulting Padé approximant for the radius $r_{2,1}$ is

$$
\begin{aligned}
& r_{2,1}=\frac{1}{9 \mathrm{e}^{-\frac{1}{2} r_{0}^{2}} b^{2}+2 r_{0}^{2}-4 b^{2}}\left\{9 \mathrm{e}^{-\frac{1}{2} b_{0}^{2}} r_{0} b^{2}-2 b^{2} t v_{0}+2 b^{2} t_{0} v_{0}+r_{0}^{2} t v_{0}-r_{0}^{2} t_{0} v_{0}-7 r_{0} b^{2}+2 r_{0}^{3}\right. \\
& \left.+\left[54 b^{4} r_{0} v_{0}\left(t-t_{0}\right) \mathrm{e}^{-\frac{1}{2} b_{0}^{2}}+4\left(\left(t-t_{0}\right)\left(b^{2}-\frac{1}{2} r_{0}^{2}\right) v_{0}-\frac{3}{2} r_{0} b^{2}\right)^{2}\right]^{\frac{1}{2}}\right\}
\end{aligned}
$$

and the velocity is

$$
\begin{gathered}
v_{2,1}=\frac{\mathrm{d} r_{2,1}}{\mathrm{~d} t}=\frac{N V G}{D V G}, \\
N V G=-\left(-27 \mathrm{e}^{-\frac{1}{2} b_{0}^{2}} r_{0} b^{4}+\left(2 b^{2}-r_{0}^{2}\right) v_{0}\left(t-t_{0}\right) r_{0}^{2}+3 r_{0} b^{2}-2 v_{0} b^{2}\left(t-t_{0}\right)\right. \\
\left.+\left\{54 b^{4} r_{0} v_{0}\left(t-t_{0}\right) \mathrm{e}^{-\frac{1}{2} r^{2}}+4\left(\left(t-t_{0}\right)\left(b^{2}-\frac{1}{2} r_{0}^{2}\right) v_{0}-3 / 2 r_{0} b^{2}\right)^{2}\right\}^{\frac{1}{2}}\right) v_{0},
\end{gathered}
$$

and

$$
D V G=\left\{54 b^{4} r_{0} v_{0}\left(t-t_{0}\right) \mathrm{e}^{-\frac{1 r_{0}^{2}}{2 b^{2}}}+4\left(\left(t-t_{0}\right)\left(b^{2}-\frac{1}{2} r_{0}^{2}\right) v_{0}-3 / 2 r_{0} b^{2}\right)^{2}\right\}^{\frac{1}{2}}\left(9 \mathrm{e}^{-\frac{1}{2} r_{0}^{2}} b^{2}+2 r_{0}^{2}-4 b^{2}\right) .
$$

\subsection{Motion with the Lane-Emden Profile}

Assuming a Lane-Emden profile, $n=5$, as given by Equation (7), the velocity is

$$
\frac{\mathrm{d} r}{\mathrm{~d} t}=\frac{N L}{D L}
$$

where

$$
N L=r_{0}^{3} v_{0}\left(3 b^{2}+r^{2}\right)^{\frac{3}{2}}\left(3 b^{2}+r_{0}^{2}\right)^{\frac{3}{2}}
$$

and

$$
D L=-3\left(3 b^{2}+r^{2}\right)^{\frac{3}{2}} \sqrt{3} r_{0}^{3} b^{3}+3\left(3 b^{2}+r_{0}^{2}\right)^{\frac{3}{2}} \sqrt{3} b^{3} r^{3}+\left(3 b^{2}+r^{2}\right)^{\frac{3}{2}}\left(3 b^{2}+r_{0}^{2}\right)^{\frac{3}{2}} r_{0}^{3} .
$$

The connected non-linear equation is

$$
\begin{aligned}
& \frac{1}{r_{0}^{3} v_{0}\left(3 b^{2}+r_{0}^{2}\right)^{\frac{3}{2}} \sqrt{3 b^{2}+r^{2}}} \times\left(5 4 ( b ^ { 2 } + \frac { 1 } { 3 } r _ { 0 } ^ { 2 } ) \left(\frac{1}{18} r_{0}^{3}\left(r-r_{0}\right) \sqrt{3 b^{2}+r^{2}}\right.\right. \\
& \left.\left.+b^{3} \sqrt{3}\left(b^{2}+\frac{1}{6} r^{2}\right)\right) \sqrt{3 b^{2}+r_{0}^{2}}-54 \sqrt{3 b^{2}+r^{2}} \sqrt{3} b^{3}\left(b^{4}+\frac{1}{2} b^{2} r_{0}^{2}+\frac{1}{18} r r_{0}^{3}\right)\right)=t-t_{0} .
\end{aligned}
$$


The Padé rational polynomial approximation of degree 2 in the numerator and degree 1 in the denominator for the left-hand side of the above equation gives

$$
\frac{N P}{2\left(3 b^{2}+r_{0}^{2}\right)^{\frac{3}{2}} v_{0}\left(2 r b^{2}-5 b^{2} r_{0}-r r_{0}^{2}\right)}=t-t_{0},
$$

where

$P N=-27\left(r-r_{0}\right)\left(-\frac{4}{9}\left(r b^{2}-\frac{5}{2} b^{2} r_{0}-\frac{1}{2} r r_{0}^{2}\right) \times\left(b^{2}+\frac{1}{3} r_{0}^{2}\right) \sqrt{3 b^{2}+r_{0}^{2}}+b^{5} \sqrt{3}\left(r-r_{0}\right)\right)$.

The Padé approximant for the radius is

$$
r_{2,1}=\frac{N R}{D R}
$$

where

$$
\begin{aligned}
N R= & -18\left(b^{2}+\frac{1}{3} r_{0}^{2}\right)^{2} b^{2}\left(-\frac{1}{2} r_{0}^{3}-\frac{1}{2} v_{0}\left(t-t_{0}\right) r_{0}^{2}\right. \\
& \left.+\frac{7}{2} b^{2} r_{0}+b^{2} v_{0}\left(t-t_{0}\right)\right) \sqrt{3 b^{2}+r_{0}^{2}}+\left(81 b^{9} r_{0}+27 b^{7} r_{0}^{3}\right) \sqrt{3} \\
& +\sqrt{972}\left(( b ^ { 2 } + \frac { 1 } { 3 } r _ { 0 } ^ { 2 } ) ^ { 4 } b ^ { 4 } \left(\frac{9}{2} \sqrt{3} r_{0} b^{5} v_{0}\left(t-t_{0}\right) \sqrt{3 b^{2}+r_{0}^{2}}\right.\right. \\
& \left.\left.+\left(-\frac{1}{2} r_{0}^{3}-\frac{1}{2} v_{0}\left(t-t_{0}\right) r_{0}^{2}-\frac{3}{2} b^{2} r_{0}+b^{2} v_{0}\left(t-t_{0}\right)\right)^{2}\left(b^{2}+\frac{1}{3} r_{0}^{2}\right)\right)\right)^{\frac{1}{2}},
\end{aligned}
$$

and

$$
D R=b^{2}\left(3 b^{2}+r_{0}^{2}\right)\left(27 b^{5} \sqrt{3}-12 b^{4} \sqrt{3 b^{2}+r_{0}^{2}}+2 b^{2} r_{0}^{2} \sqrt{3 b^{2}+r_{0}^{2}}+2 r_{0}^{4} \sqrt{3 b^{2}+r_{0}^{2}}\right),
$$

and the velocity is

$$
v_{2,1}=\frac{\mathrm{d} r_{2,1}}{\mathrm{~d} t}=\frac{N V L}{D V L}
$$

where

$$
\begin{aligned}
N V L= & -18 \sqrt{3}\left(3 b^{2}+r_{0}^{2}\right) v_{0}\left(-243\left(b^{2}+\frac{1}{3} r_{0}^{2}\right)^{2} b^{7} r_{0} \sqrt{3}\right. \\
& +\sqrt{972}\left\{( b ^ { 2 } + \frac { 1 } { 3 } r _ { 0 } ^ { 2 } ) ^ { 4 } b ^ { 4 } \left(9 / 2 \sqrt{3} r_{0} b^{5} v_{0}\left(t-t_{0}\right) \sqrt{3 b^{2}+r_{0}^{2}}\right.\right. \\
& \left.\left.+\left(b^{2}+\frac{1}{3} r_{0}^{2}\right)\left(-\frac{1}{2} r_{0}^{3}-\frac{1}{2} v_{0}\left(t-t_{0}\right) r_{0}^{2}-3 / 2 b^{2} r_{0}+b^{2} v_{0}\left(t-t_{0}\right)\right)^{2}\right)\right\}^{\frac{1}{2}} \\
& \left.\times\left(2 b^{2}-r_{0}^{2}\right)\right) \sqrt{3 b^{2}+r_{0}^{2}}-108\left(b^{2}+\frac{1}{3} r_{0}^{2}\right)^{3} b^{2}\left(-1 / 2 r_{0}^{3}-\frac{1}{2} v_{0}\left(t-t_{0}\right) r_{0}^{2}\right. \\
& \left.-3 / 2 b^{2} r_{0}+b^{2} v_{0}\left(t-t_{0}\right)\right)\left(b^{2}-\frac{1}{2} r_{0}^{2}\right),
\end{aligned}
$$

and 


$$
\begin{aligned}
D V L= & 18 \sqrt{972} \sqrt{3}\left\{( b ^ { 2 } + \frac { 1 } { 3 } r _ { 0 } ^ { 2 } ) ^ { 4 } b ^ { 4 } \left(9 / 2 \sqrt{3} r_{0} b^{5} v_{0}\left(t-t_{0}\right) \sqrt{3 b^{2}+r_{0}^{2}}\right.\right. \\
& \left.\left.+\left(b^{2}+\frac{1}{3} r_{0}^{2}\right)\left(-\frac{1}{2} r_{0}^{3}-\frac{1}{2} v_{0}\left(t-t_{0}\right) r_{0}^{2}-3 / 2 b^{2} r_{0}+b^{2} v_{0}\left(t-t_{0}\right)\right)^{2}\right)\right\}^{\frac{1}{2}} \\
& \times\left(\left(-12 b^{4}+2 b^{2} r_{0}^{2}+2 r_{0}^{4}\right) \sqrt{3 b^{2}+r_{0}^{2}}+27 b^{5} \sqrt{3}\right) .
\end{aligned}
$$

\section{Astrophysical Applications}

In the previous section, we derived three equations of motion in the form of non-linear equations and three Padé approximated equations of motion. We now check the reliability of the numerical and approximated solutions on four SNRs: Tycho, see [8], Cas A, see [9], Cygnus loop, see [10], and SN 1006, see [11]. The three astronomical measurable parameters are the time since the explosion in years, $t$, the actual observed radius in $\mathrm{pc}, r$, and the present velocity of expansion in $\mathrm{km} \cdot \mathrm{s}^{-1}$, see Table 1 . The astrophysical units have not yet been specified: pc for length and yr for time are the units most commonly used by astronomers. With these units, the initial velocity is $v_{0}\left(\mathrm{~km} \cdot \mathrm{s}^{-1}\right)=9.7968 \times 10^{5} v_{0}\left(\mathrm{pc} \cdot \mathrm{yr}^{-1}\right)$. The determination of the four unknown parameters, which are $t_{0}, r_{0}, v_{0}$ and $b$, can be obtained by equating the observed astronomical velocities and radius with those obtained with the Padé rational polynomial, i.e.

$$
\begin{gathered}
r_{2,1}=\operatorname{Radius}(p c), \\
v_{2,1}=\operatorname{Velocity}\left(\mathrm{km} \cdot \mathrm{s}^{-1}\right) .
\end{gathered}
$$

In order to reduce the unknown parameters from four to two, we fix $v_{0}$ and $t_{0}$. The two parameters $b$ and $r_{0}$ are found by solving the two non-linear Equations (38) and (39). The results for the three types of profiles here adopted are reported in Tables 2-4.

Table 1. Observed astronomical parameters of SNRs.

\begin{tabular}{ccccc}
\hline Name & Age $(\mathrm{yr})$ & Radius $(\mathrm{pc})$ & Velocity $\left(\mathrm{km} \cdot \mathrm{s}^{-1}\right)$ & References \\
\hline Tycho & 442 & 3.7 & 5300 & Williams et al. 2016 \\
Cas A & 328 & 2.5 & 4700 & Patnaude and Fesen 2009 \\
Cygnus loop & 17,000 & 24.25 & 250 & Chiad et al. 2015 \\
SN 1006 & 1000 & 10.19 & 3100 & Uchida et al. 2013 \\
\hline
\end{tabular}

Table 2. Theoretical parameters of SNRs for the padé approximated equation of motion with an exponential profile.

\begin{tabular}{ccccccc}
\hline Name & $t_{0}(\mathrm{yr})$ & $r_{0}(\mathrm{pc})$ & $v_{0}\left(\mathrm{~km} \cdot \mathrm{s}^{-1}\right)$ & $b(\mathrm{pc})$ & $\delta(\%)$ & $\Delta v\left(\mathrm{~km} \cdot \mathrm{s}^{-1}\right)$ \\
\hline Tycho & 0.1 & 1.203 & 8000 & 0.113 & 5.893 & -1.35 \\
Cas A & 1 & 0.819 & 8000 & 0.1 & 6.668 & -3.29 \\
Cygnus loop & 10 & 12.27 & 3000 & 45.79 & 6.12 & -0.155 \\
SN 1006 & 1 & 5.49 & 3100 & 2.332 & 1.455 & -12.34 \\
\hline
\end{tabular}


Table 3. Theoretical parameters of SNRs for the Padé approximated equation of motion with a gaussian profile.

\begin{tabular}{ccccccc}
\hline Name & $t_{0}(\mathrm{yr})$ & $r_{0}(\mathrm{pc})$ & $v_{0}\left(\mathrm{~km} \cdot \mathrm{s}^{-1}\right)$ & $b(\mathrm{pc})$ & $\delta(\%)$ & $\Delta v\left(\mathrm{~km} \cdot \mathrm{s}^{-1}\right)$ \\
\hline Tycho & 0.1 & 1.022 & 8000 & 0.561 & 8.517 & -10.469 \\
Cas A & 1 & 0.741 & 7000 & 0.406 & 7.571 & -13.16 \\
Cygnus loop & 10 & 11.92 & 3000 & 21.803 & 7.875 & -0.161 \\
SN 1006 & 1 & 5.049 & 10000 & 4.311 & 4.568 & -18.58 \\
\hline
\end{tabular}

Table 4. Theoretical parameters of SNRs for the Padé approximated equation of motion with a Lane-Emden profile.

\begin{tabular}{ccccccc}
\hline Name & $t_{0}(\mathrm{yr})$ & $r_{0}(\mathrm{pc})$ & $v_{0}\left(\mathrm{~km} \cdot \mathrm{s}^{-1}\right)$ & $b(\mathrm{pc})$ & $\delta(\%)$ & $\Delta v\left(\mathrm{~km} \cdot \mathrm{s}^{-1}\right)$ \\
\hline Tycho & 0.1 & 0.971 & 8000 & 0.502 & 3.27 & -14.83 \\
Cas A & 1 & 0.635 & 8000 & 0.35 & 4.769 & -23.454 \\
Cygnus loop & 10 & 11.91 & 3000 & 27.203 & 7.731 & -0.162 \\
SN 1006 & 1 & 5 & 10000 & 4.85 & 3.297 & -19.334 \\
\hline
\end{tabular}

The goodness of the approximation is evaluated through the percentage error, $\delta$, which is

$$
\delta=\frac{\left|r_{2,1}-r_{E}\right|}{r_{E}} \times 100,
$$

where $r_{2,1}$ is the Padé approximated radius and $r_{E}$ is the exact solution which is obtained by solving numerically the non-linear equation of motion, as an example Equation (12) in the exponential case. The numerical values of $\delta$ are reported in column 6 of Tables 2-4. Another useful astrophysical variable is the predicted decrease in velocity on the basis of the Padé approximated velocity, $v_{2,1}$, in 10 years, see column 7 of Tables 2-4.

\section{Conclusion}

The expansion of an SNR can be modeled by the conservation of momentum in the presence of a decreasing density: here we analysed an exponential, a Gaussian and a Lane-Emden profile. The three equations of motion have complicated left-hand sides but simple left-hand sides, viz., $\left(t-t_{0}\right)$. The application of the Padé approximant to the left-hand side of the complicated equation of motion allows finding three approximate laws of motion, see Equations $(14,23,32)$, and three approximate velocities, see Equations $(15,24,35)$. The astrophysical test is performed on four spherical SNRs assumed to be spherical and the four sets of parameters are reported in Tables 2-4. The percentage of error of the Padé approximated solutions for the radius is always less than $10 \%$ with respect to the numerical exact solution, see column 6 of the three last tables. In order to produce an astrophysical prediction, the theoretical decrease in velocity for the four SNRs here analysed is evaluated, see column 7 of Tables 2-4. 


\section{References}

[1] Sedov, L.I. (1959) Similarity and Dimensional Methods in Mechanics. Academic Press, New York.

[2] Dyson, J.E. and Williams, D.A. (1997) The Physics of the Interstellar Medium. Institute of Physics Publishing, Bristol. https://doi.org/10.1887/075030460X

[3] McCray, R.A. (1987) Coronal Interstellar Gas and Supernova Remnants. In: Dalgarno, A. and Layzer, D., Eds., Spectroscopy of Astrophysical Plasmas, Cambridge University Press, Cambridge, 255-278. https://doi.org/10.1017/CBO9780511564659.011

[4] Zaninetti, L. (2011) Time-Dependent Models for a Decade of SN 1993J. Astrophysics and Space Science, 333, 99. https://doi.org/10.1007/s10509-011-0609-X

[5] Olver, F.W.J., Lozier, D.W., Boisvert, R.F. and Clark, C.W. (2010) NIST Handbook of Mathematical Functions. Cambridge University Press, Cambridge.

[6] Lane, H.J. (1870) On the Theoretical Temperature of the Sun, under the Hypothesis of a Gaseous Mass Maintaining Its Volume by Its Internal Heat, and Depending on the Laws of Gases as Known to Terrestrial Experiment American Journal of Science, 148, 57. https://doi.org/10.2475/ajs.s2-50.148.57

[7] Emden, R. (1907) Gaskugeln: Anwendungen der mechanischen warmetheorie auf kosmologische und meteorologische Probleme. B. Teubner, Berlin.

[8] Williams, B.J., Chomiuk, L., Hewitt, J.W., Blondin, J.M., Borkowski, K.J., Ghavamian, P., Petre, R. and Reynolds, S.P. (2016) An X-Ray and Radio Study of the Varying Expansion Velocities in Tycho Supernova Remnant. ApJ, 823, L32.

[9] Patnaude, D.J. and Fesen, R.A. (2009) Proper Motions and Brightness Variations of Nonthermal X-Ray Filaments in the Cassiopeia A Supernova Remnant. ApJ, 697, 535.

[10] Chiad, B.T., Ali, L.T. and Hassani, A.S. (2015) Determination of Velocity and Radius of Supernova Remnant after 1000 yrs of Explosion. International Journal of Astronomy and Astrophysics, 5, 125.

[11] Uchida, H., Yamaguchi, H. and Koyama, K. (2013) Asymmetric Ejecta Distribution in SN 1006. ApJ, 771, 56.

Submit or recommend next manuscript to SCIRP and we will provide best service for you:

Accepting pre-submission inquiries through Email, Facebook, LinkedIn, Twitter, etc.

A wide selection of journals (inclusive of 9 subjects, more than 200 journals)

Providing 24-hour high-quality service

User-friendly online submission system

Fair and swift peer-review system

Efficient typesetting and proofreading procedure

Display of the result of downloads and visits, as well as the number of cited articles

Maximum dissemination of your research work

Submit your manuscript at: http://papersubmission.scirp.org/

Or contact jhepgc@scirp.org 